\title{
The Extended Dwell Peripheral Intravenous Catheter Is an Alternative Method of NICU Intravenous Access
}

\author{
Kimberlee B. Chenoweth, DNP; Jia-Wen Guo, PhD, RN; Belinda Chan, MD
}

\begin{abstract}
Background: Establishing vascular access is a common neonatal intensive care unit procedure. The extended dwell peripheral intravenous (EPIV) catheter is a 6-cm and 8-cm silicone catheter for peripheral vein insertion, which is a newer vascular access device than peripherally inserted central catheters (PICCs) and peripheral intravenous (PIV) catheter. Extended dwell peripheral intravenous catheters have been widely used in adults but evidence in neonates is lacking. Purpose: To explore indwell time, success rate, catheter-associated complications, and cost among EPIV catheters, PICCs, and PIV catheters in neonates.

Methods: We retrospectively compare patient demographics, indwell time, success rate, and catheter-associated complications, and analyze the rate of hyaluronidase-treated intravenous (IV) fluid extravasation on neonates who had an EPIV catheter, a PICC, or a PIV catheter in a level III neonatal intensive care unit. We also estimate the insertion cost of these 3 vascular access devices on the basis of our hospital charges.

Results: Extended dwell peripheral intravenous catheters were inserted in 432 neonates with an indwell time of $4.0 \pm$ 2.3 (mean \pm SD) days. Peripherally inserted central catheters were inserted in 202 neonates with an average indwell time of $7.3 \pm 4.4$ (mean \pm SD) days, which was longer than EPIV catheters $(P<.001)$. Peripherally inserted central catheters had a higher success rate of $83.6 \%$ than $71.7 \%$ of EPIV catheters, meaning succeeded in lasting through the completion of therapy $(P=.001)$. Peripherally inserted central catheters were associated with 4 cases of life-threatening complications; none was seen in the EPIV catheter group. The incidence of hyaluronidase-treated IV fluid extravasation was less in EPIV catheter recipients (1.2\%) than in the PIV catheter recipients (3.9\%) $(P=.004)$; none was in the PICC group. Cost savings were noted with using an EPIV catheter.

Implications for Practice: Extended dwell peripheral intravenous catheter is a feasible option for neonatal vascular access.

Implications for Research: These data provide a baseline for future studies to explore the efficacy and effectiveness of EPIV catheter in the neonates.

Key Words: catheter-associated complications, cost, extended dwell peripheral intravenous catheter, indwell time, midline intravenous catheter, neonates, peripheral venous catheter, peripherally inserted central catheter, placement success rate, vascular access
\end{abstract}

\section{BACKGROUND/SIGNIFICANCE}

Vascular access is often needed in neonatal intensive care unit (NICU) neonates. The majority of NICU neonates require vascular access during their stay for prolonged nutritional support and medications.

\footnotetext{
Author Affiliations: Primary Children's Neonatal Nurse Practitioner Group, Intermountain Healthcare, Salt Lake City, Utah (Dr Chenoweth); College of Nursing, University of Utah, Salt Lake City, Utah (Dr Guo); and Division of Neonatology, Department of Pediatrics, University of Utah School of Medicine, Salt Lake City, Utah (Dr Chan).

The authors declare no conflicts of interest.

This is an open-access article distributed under the terms of the Creative Commons Attribution-Non Commercial-No Derivatives License 4.0 (CCBY-NC-ND), where it is permissible to download and share the work provided it is properly cited. The work cannot be changed in any way or used commercially without permission from the journal.

Correspondence: Kimberlee B. Chenoweth, DNP, Primary Children's Neonatal Nurse Practitioner Group, Intermountain Healthcare, 100 Mario Capecchi Dr, Salt Lake City, UT 84113 (kimberlee.chenoweth@imail.org). Copyright (C) 2018 The Authors. Published by Wolters Kluwer Health, Inc. on behalf of the National Association of Neonatal Nurses
}

DOI: 10.1097/ANC.0000000000000515
Establishing vascular access is challenging in NICU neonates due to their small and extremely fragile veins. ${ }^{1}$ The 2 most commonly used neonatal vascular access devices are peripheral intravenous (PIV) catheter and peripherally inserted central catheter (PICC). However, both devices have well-known limitations and risks. ${ }^{2}$ The neonatal extended dwell peripheral intravenous (EPIV) catheter is a new device that became available in recent years and seems to have advantages over the PIV catheter and PICC, but comparative effectiveness studies have not been reported from the NICU population. ${ }^{3}$

A PICC is an intravenous catheter that is inserted peripherally and threads into the central venous circulation and is made of silicone, polyurethane, or polyethylene. ${ }^{4}$ To be considered centrally placed, the tip of the PICC should be in the superior or inferior vena cava ${ }^{5}$ (Figure 1). It is intended for long-term nutrition and medication therapies as it can remain in place for months at a time. Peripherally inserted central catheter requires additionally trained nurses for insertion and radiographs to confirm correct 


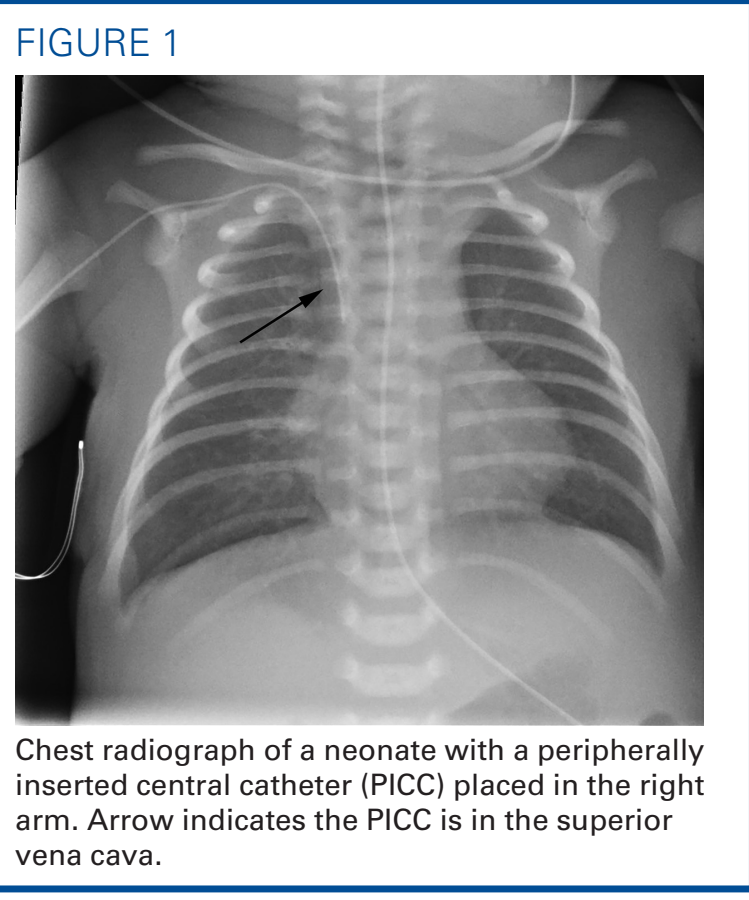

placement. ${ }^{5}$ Peripherally inserted central catheter can be associated with complications including central line-associated bloodstream infection (CLABSI), phlebitis, infiltration, occlusion, catheter leakage leading to pleural effusion and peritonitis, cardiac arrhythmia, pericardial effusion, and cardiac tamponade. ${ }^{6}$ Peripherally inserted central catheterrelated cases of CLABSI and pericardial effusion had been directly linked to neonatal death. ${ }^{6-8}$

The most widely used vascular access device in the NICU is a PIV catheter, which is commonly a 22- or 24-gauge Teflon radiopaque over the needle catheter. 9 A PIV catheter is easier to place than a central catheter, but it does not last as long and can often take multiple attempts to be successfully placed. ${ }^{1,10-12}$ Most NICU nurses are competent at inserting a PIV catheter, which is usually placed in the extremities or the scalp. ${ }^{9}$ Despite the high rate of use and long history, PIV catheter still has a poor track record. One study $(\mathrm{n}=72)$ among the neonatal population showed that the average length of time a PIV catheter was in place before complications required removal was 30 hours, ${ }^{13}$ while another larger study $(\mathrm{n}=250)$ among the neonatal population showed 37 hours to be the average length of time. ${ }^{14}$ This is an extremely short period compared with the length of nutritional supplementation and medication needed in most NICU neonates. Approximately $95 \%$ of PIV catheters are removed before the completion of therapy generally due to complications such as infiltration, infection, clotting, or other problems. ${ }^{9}$ Infiltration rate among neonates is $57 \%$ to $70 \%$ and tissue damage from extravasation occurs in $11 \%$ to $25 \% .{ }^{15}$ Extravasation can lead to

\section{What This Study Adds}

- A retrospective analysis of the pros and cons of EPIV catheters, PIV catheters, and PICCs within the neonatal population, which is a step toward a larger, randomized control trial of neonatal vascular access.

- Information that allows the NICU clinician to make a more informed decision regarding the use of vascular access devices.

tissue necrosis, infection, limb disfigurement, and functional loss. ${ }^{16}$ Treatments vary among each unit protocol including immediate line removal, limb elevation, saline washout, and thermal compress. ${ }^{16}$ Subcutaneous injection of hyaluronidase is one of the pharmaceutical treatments of IV extravasation. Hyaluronidase is an enzyme that helps absorb and dispense extravasated irritants, which has shown to reduce the severity of tissue damage. ${ }^{17}$

Extended dwell peripheral intravenous catheter, which is also known as a midline catheter, has been widely used in adults since the 1950s, but there is very limited information available regarding its use in the NICU population. ${ }^{3}$ In adults, EPIV catheter has been showed to reduce cannulation attempts and improve patient satisfaction and hospital efficiency; however, it is associated with complications such as phlebitis and thrombosis. ${ }^{18,19}$ Neonatal EPIV catheter was first introduced in $1992^{20}$; its design has been evolving and improving since then. The latest generation of neonatal EPIV catheter is a short single lumen silicon catheter, manufactured as either a $6 \mathrm{~cm}$ or an $8 \mathrm{~cm}$ length for neonates (length used on the basis of neonate's size or unit preference), which is designed to remain intravenously for up to 29 days. ${ }^{21}$ Typically, the catheter is inserted into a peripheral vein on the forearm or leg, with the catheter tip located below the axilla of the arm (Figures 2A and B) or below the groin of the leg. ${ }^{6}$ Extended dwell peripheral intravenous catheter placement does not require $\mathrm{x}$-ray film confirmation unless there is difficulty in insertion, advancement, or flushing. ${ }^{22}$ According to the Infusion Nurses Society's standards of practice, EPIV catheter is used as a peripheral vascular access which should not be used for "continuous vesicant therapy, parenteral nutrition, or infusates with an osmolality greater than $900 \mathrm{mOsm} / \mathrm{L} " 22$ including dextrose greater than $12.5 \%$ IV fluid, total parenteral nutrition greater than $900 \mathrm{mOsm} / \mathrm{L}$, vasopressors, chemotherapy, sodium chloride greater than $3 \%$, and sodium bicarbonate. ${ }^{23}$

Limited research points to the benefit of EPIV catheter over conventional PIV catheter for neonates because of the higher percentage of EPIV catheter staying in until the end of treatment and its comparative longer dwell time. Lesser et $\mathrm{al}^{12}$ reported the use of EPIV catheters on 9 very low birth-weight (VLBW) infants, in which EPIV catheters had a longer indwell time than PIV catheters (9 vs 3 days). 

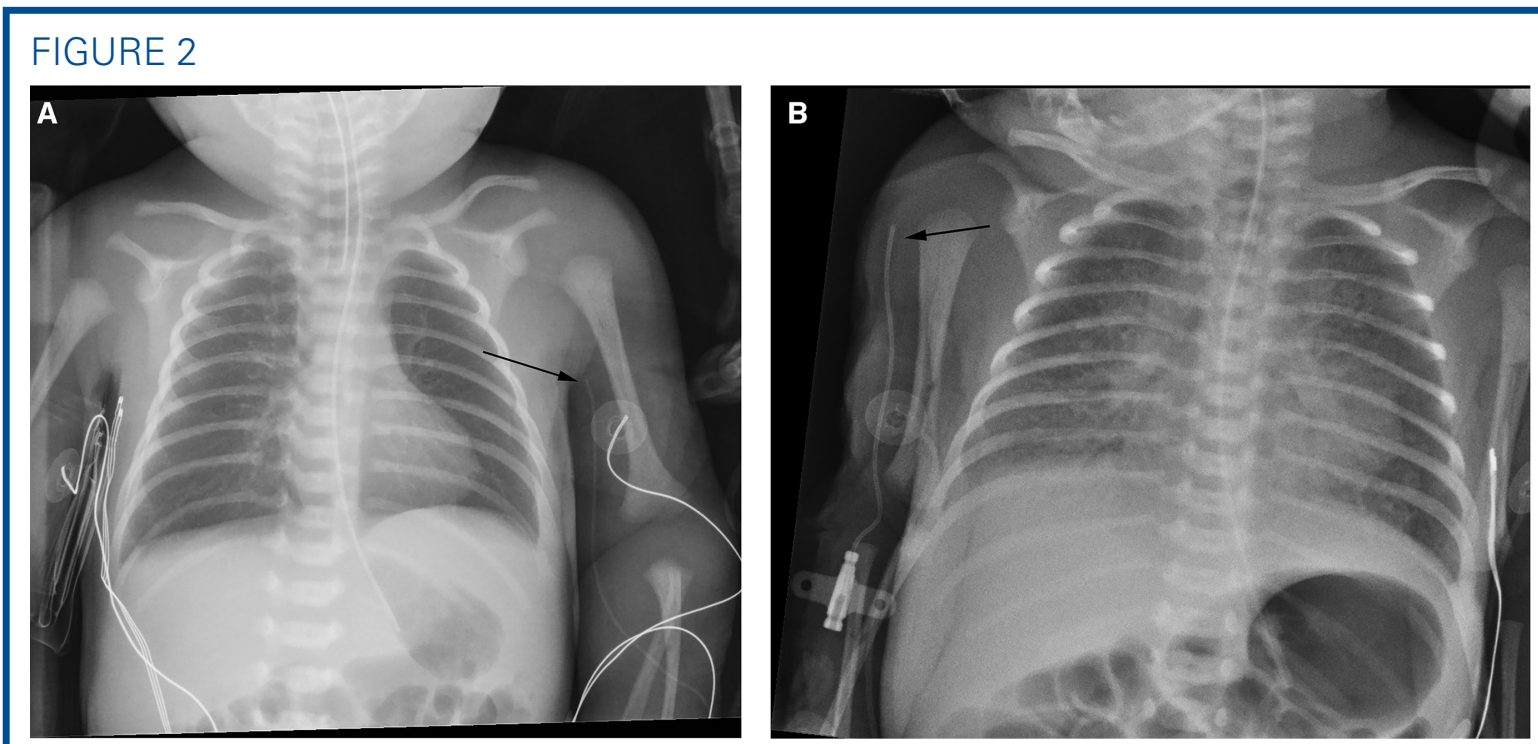

(A) Chest radiograph of a neonate with an extended dwell peripheral intravenous (EPIV) catheter placed in the left arm. Arrow indicates the tip of the EPIV catheter in the left brachial vein below the left shoulder. (B) Chest radiograph of a neonate with an EPIV catheter placed in the right arm. Arrow indicates the tip of the EPIV catheter in the right cephalic vein below the right shoulder.

Similarly, Wyckoff $(\mathrm{n}=143 \text { EPIV catheters })^{1}$ and Dawson ${ }^{10}$ had separately suggested that EPIV catheter was associated with longer indwell time and fewer cannulation attempts than PIV catheter in neonates. Leick-Rude and Haney ${ }^{11}$ conducted a nonrandomized prospective study of 1130 EPIV catheters used in 858 neonates, who were ranging from 360 to $800 \mathrm{~g}$ in weights and 23 to 42 weeks of gestational age at birth. It has shown that the average indwell time for EPIV catheters was 8.7 days, with a success rate of $57 \%$ meaning the catheters staying in until the end of treatment. ${ }^{11}$

In 2012, we devised and implemented the EPIV catheter protocol in our level III NICU. Along the process, we compiled detailed data regarding our EPIV catheter experience. As a step toward building an evidence-based EPIV catheter NICU practice, the purpose of this study was to explore indwell time, success rate, catheter-associated complications, and insertion cost among EPIV catheter, PICC, and PIV catheter in our NICU. This information may allow NICU clinicians to make a more informed decision regarding the choices of vascular access devices in neonates.

\section{METHODS}

This retrospective study was conducted to compare patient demographics, indwell time, placement success rate, and catheter-associated complications among neonates who had an EPIV catheter, a PICC, or a PIV catheter in a level III NICU. Data regarding
IV fluid extravasation rate were collected on neonates with EPIV catheter, PICC, and PIV catheter. Insertion cost of these 3 vascular access options was estimated on the basis of our unit charges.

\section{Settings}

This study was conducted at a level III NICU in the Intermountain Medical Center at Salt Lake City, Utah, which is a 48-bed unit that provided care for more than 3000 admitted complex term and preterm neonates during the time frame of the study.

\section{Sample}

Between August 2012 and December 2016, 3526 neonates were admitted to the Intermountain Medical Center NICU. A retrospective review was performed on all neonates who were 32 weeks of gestation or older and weighed $1500 \mathrm{~g}$ or more at birth with EPIV catheter, PICC, and/or PIV catheter placements. In this study period, there were 2828 neonates who met the gestational age (GA) and birth weight (BW) inclusion criteria; all of them had PIV catheters inserted during their hospitalization. In addition to PIV catheters, 202 of these neonates required PICC placement and 432 of these neonates required EPIV catheter placement.

\section{Measures}

In our NICU, data sheets were created for all neonates, with each EPIV catheter and PICC insertion containing patient demographics and catheterrelated data. The data collected for this study included GA at birth, BW, the reason for insertion, 
numbers of insertion attempts, indwell time, the reason for removal due to completion of treatment, and complications in neonates with EPIV catheter and PICC. Data on PIV catheter insertion were not separately documented in the same extensive details as EPIV catheter or PICC insertion in our unit. With the retrospective nature of this study, we were unable to practically perform chart reviews on more than 2800 neonates who had PIV catheters during their hospitalization and to compare their demographics, indwell time, and success rate with EPIV catheter and PICC groups.

Hyaluronidase is the treatment of choice in our unit for neonates with significant IV fluid extravasation. Based on our pharmacy database, we studied the number of hyaluronidase doses used in neonates, which was used to compute the incidence of hyaluronidase-treated IV fluid extravasation associated with each type of catheter. Insertion cost of EPIV catheter, PICC, and PIV catheter was calculated on the basis of 3 categories: (1) direct supply costs, (2) labor costs, and (3) radiographic charges in Utah.

\section{Statistics}

Descriptive statistics was used to summarize and describe the numeric data such as GA and BW. The Mann-Whitney $U$ test was used to assess differences in BW, GA, and indwell time between groups (eg, EPIV catheter, PICC); for easier understanding, we presented mean and standard deviation to describe the variables and the $P$ values based on the MannWhitney $U$ tests in Tables 1 and 2 . The $\chi^{2}$ test was used to assess differences in success rate and incidence of hyaluronidase-treated IV extravasation. All the descriptive and statistical analyses were made using the SPSS 24 for Windows and using a significance level of .050.

\section{Procedure}

Extended dwell peripheral intravenous catheter is a relatively new innovation having been available for neonates. With the knowledge gained through the literature review, our NICU RN vascular access team introduced the EPIV catheter product and developed the EPIV catheter protocol in 2012. The EPIV catheter used was a $1.9 \mathrm{Fr} \times 6-\mathrm{cm}$ and a 1.9 Fr $\times 8$-cm silicone-based catheter ${ }^{21}$ (Figure 3). Candidates for EPIV catheter insertion are selected by the healthcare team on the basis of the need for multiple days of IV antibiotics and/or IV nutrition. Inclusion criteria for EPIV catheter insertion include neonates who are 32 weeks of gestation or more and weighing 1500 or more at birth with difficult or limited venous access that is likely to be required up to 4 weeks. Neonates requiring fluid greater than dextrose $12.5 \%$ concentration, total parenteral nutrition osmolarity greater than $900 \mathrm{mOsm} / \mathrm{L}$, and/or medications that are administrated via central catheters are excluded from receiving an EPIV catheter. Peripherally inserted central catheter or umbilical venous catheter is inserted in neonates who require central vascular access. Peripheral intravenous catheter is routinely the first vascular access placed on critically ill neonates admitted to NICU.

The data collection occurred after the privacy board of the Intermountain Healthcare Institutional Review Board and The University of Utah Institutional Review Board approved the study protocol. The privacy board granted a waiver from individual parental consent because this was a deidentified data-only retrospective study with appropriate privacy protection.

\section{RESULTS}

Between August 2012 and December 2016, 3526 neonates were admitted to the Intermountain Medical Center NICU. During this time, EPIV catheters were inserted in 432 neonates who were born at 32 to 41 weeks of GA ( $35 \pm 3$ weeks, mean \pm SD), with BW 1500 to $4400 \mathrm{~g}(2700 \pm 700 \mathrm{~g}$, mean \pm SD) (Table 1). Total EPIV catheter days were 1735, with a mean indwell time of $4.0 \pm 2.3$ days (ranging 1-29 days). A portion of EPIV catheters $(13.3 \%)$ was inserted less than 2 days prior to the completion of therapy. In neonates with an EPIV catheter, $71.7 \%$ of catheters succeeded in lasting through the completion of therapy (Table 2). The others $(28.3 \%)$ were removed before the completion of therapy because the catheters had failed. The reasons for failures were leaking $(\mathrm{n}=37,8.6 \%)$, infiltration $(\mathrm{n}=$ $29,6.7 \%)$, palpable venous cord or hardening ( $\mathrm{n}=$ $18,4.2 \%)$, clotting $(\mathrm{n}=15,3.5 \%)$, accidental dislodgement $(n=14,3.2 \%)$, or other reasons $(n=8$, $1.8 \%$ ) including sluggish when flushing, redness, swelling, phlebitis, broken catheter, or used more

\begin{tabular}{|c|c|c|c|c|}
\hline & $\begin{array}{l}\text { EPIV Catheter } \\
\quad(n=432)\end{array}$ & $\operatorname{PICC}(n=202)$ & $\begin{array}{l}\text { PIV Catheter } \\
\text { (n= 2828) }\end{array}$ & $\boldsymbol{P}$ \\
\hline Gestational age at birth, mean (SD), wk & $35.8(2.6)$ & $36.5(2.7)$ & $\ldots$ & .002 \\
\hline Weight at birth, mean (SD), g & $2687(731.7)$ & $2820(756.7)$ & $\ldots$ & .200 \\
\hline
\end{tabular}




\begin{tabular}{|c|c|c|c|c|}
\hline & $\begin{array}{l}\text { EPIV Catheter } \\
\quad(n=432)\end{array}$ & $\begin{array}{c}\text { PICC } \\
(n=202)\end{array}$ & $\begin{array}{c}\text { PIV } \\
\text { Catheter } \\
(\mathbf{n}=\mathbf{2 8 2 8})\end{array}$ & $P$ \\
\hline \multicolumn{5}{|l|}{ Placement success } \\
\hline Indwell time, mean (SD), d & $4.0(2.3)$ & $7.31(4.4)$ & ... & $<.001^{a}$ \\
\hline Success rate, $\%$ & 71.7 & 83.6 & $\ldots$ & $.001^{\mathrm{b}}$ \\
\hline \multicolumn{5}{|l|}{ Complications } \\
\hline $\begin{array}{l}\text { Life-threatening complication } \\
\text { (cases per } 1000 \text { catheter } \\
\text { days) }\end{array}$ & $\cdots$ & $\begin{array}{l}\text { CLABSI }(0.68 / 1000) \\
\text { Premature ventricular } \\
\text { contraction }(0.68 / 1000) \\
\text { Superior vena cava } \\
\text { obstruction }(0.68 / 1000) \\
\text { Peritonitis }(0.68 / 1000)\end{array}$ & $\cdots$ & \\
\hline $\begin{array}{l}\text { Incidence of hyaluronidase- } \\
\text { treated IV fluid } \\
\text { extravasation, } \%\end{array}$ & 1.2 & $\cdots$ & 3.9 & $.004^{\mathrm{b}}$ \\
\hline \multicolumn{5}{|l|}{ Insertion cost (for 1-wk use) ${ }^{c}$} \\
\hline Supplies & $\$ 43.15$ & $\$ 120.38$ & $\$ 32.67$ & \\
\hline Labor & $\$ 30.00$ & $\$ 60.00$ & $\$ 67.50$ & \\
\hline Radiography & $\$ 0$ & $\$ 410.00$ & $\$ 0$ & \\
\hline Total & $\$ 73.15$ & $\$ 590.38$ & $\$ 100.17$ & \\
\hline \multicolumn{5}{|c|}{$\begin{array}{l}\text { Abbreviations: CLABSI, central line-associated bloodstream infection; EPIV, extended dwell peripheral intravenous; IV, intravenous; PICC, } \\
\text { peripherally inserted central catheter; PIV, peripheral intravenous; PVC, Premature ventricular contraction. } \\
{ }^{a} \text { The statistical outcome of Mann-Whitney U test. } \\
\text { bThe statistical outcome of } \chi^{2} \text { test. } \\
{ }^{\circ} \text { Cost is based on Intermountain Medical Center charges in Utah. }\end{array}$} \\
\hline
\end{tabular}

than 29 days as recommended by the manufacturer. We found no statistical significance in BW or GA $(P=.17)$ comparing neonates with EPIV catheters that lasted with those that failed early.

During this time, PICCs were inserted in 202 neonates who were 32 weeks of GA or more $(36 \pm 3$ weeks, mean \pm SD) at birth and weighing $1500 \mathrm{~g}$ $\mathrm{BW}$ or more $(2800 \pm 800 \mathrm{~g}$, mean $\pm \mathrm{SD})($ Table 1$)$.

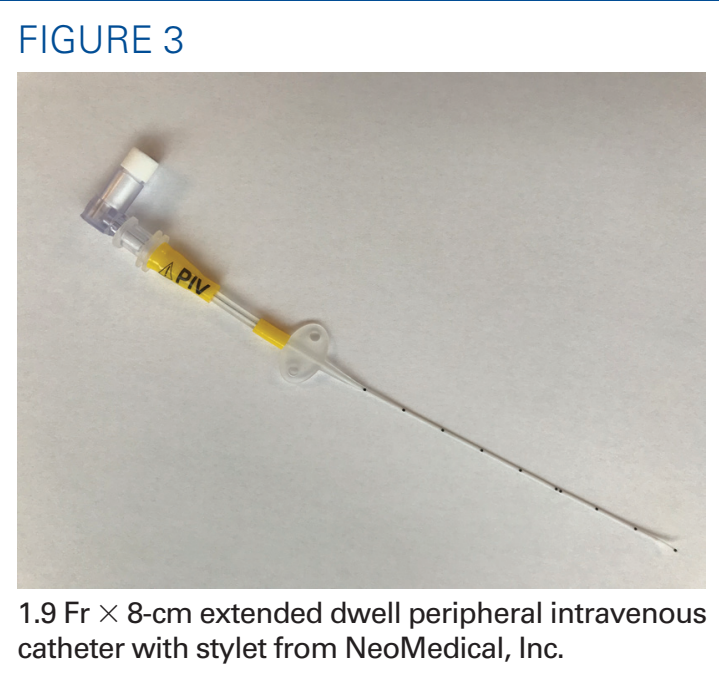

Peripherally inserted central catheter group was 1 week older in GA at birth than the EPIV catheter group $(P=.002)$. Total PICC catheter days were 1476 , with a mean indwell time of $7.3 \pm 4.4$ days, which was longer than EPIV catheter indwell days $(P<.001)$. The success rate of PICC was $83.6 \%$, which was a higher success rate than EPIV catheter $(71.7 \%)(P=.001)$ (Table 2).

Catheter-associated life-threatening complications are shown in Table 2. Peripherally inserted central catheters were associated with 1 case of each of the following: CLABSI (0.68/1000 catheter days), premature ventricular contractions $(0.68 / 1000$ catheter days), superior vena cava obstruction $(0.68 / 1000$ catheter days), and peritonitis $(0.68 / 1000$ catheter days). No life-threatening complications were seen in 1735 EPIV catheter days.

In the EPIV catheter group of 432 neonates, we identified 29 cases of IV infiltration and 5 of those significant cases were treated with hyaluronidase $(5 / 432,1.2 \%)$ (Table 2$)$. During the same period, 2828 neonates who were born at 32 weeks of GA or more and weighing $1500 \mathrm{~g}$ or more BW were admitted to the NICU and had a PIV catheter inserted. Among those, 110 neonates were treated with hyaluronidase for PIV catheter-associated IV fluid 
extravasation injury. A higher rate of significant extravasation $(110 / 2828,3.9 \%)$ requiring treatment was observed in the PIV catheter group than in the EPIV catheter group $(P=.004)$ (Table 2). No hyaluronidase was used in neonates with PICCs.

Insertion cost of 1-week use of EPIV catheter, PICC, and PIV catheter was estimated on the basis of the Intermountain Medical Center supply charge, hourly nursing wages, and $\mathrm{x}$-ray film charges (Table 2). Peripheral intravenous catheter, EPIV catheter, and PICC supplies cost \$7.26, \$43.15, and $\$ 120.38$ each, respectively. As an average, PIV catheter is replaced every 1.5 days, or 4.5 PIV catheters in 7 days, ${ }^{19} 1$-week PIV catheter supply costs a total of $(\$ 7.26 \times 4.5) \$ 32.67$. Inserting PICCs is more labor-intensive and time-consuming than inserting EPIV catheters, as they require precise catheter tip location with $\mathrm{x}$-ray films confirmation. Labor cost is calculated on the basis of average nursing wages of $\$ 30$ per hour. Two nurses are typically needed to insert EPIV catheter and PICC, requiring 30 minutes and 60 minutes each, with an estimated labor cost of $\$ 30$ and \$60, respectively. Each PIV catheter placement with 2 nurses assisting typically takes less than 15 minutes; however, labor cost accumulates up to $\$ 67.5$ for placing 4.5 PIV catheters over 1 week. An average of 2 radiographs ( $\$ 410$ each) is needed to confirm appropriate placement of PICC; none is necessary for EPIV catheter and PIV catheter. As a result, 1-week costs of EPIV catheter were \$517 and \$27 less than PICC and PIV catheter.

\section{DISCUSSION}

Extended dwell peripheral intravenous catheter has been available for more than a decade and has proven valuable for certain circumstances in adult patients. ${ }^{24}$ However, a rigorous assessment of its indwell time, placement success rate, safety, and cost in a NICU setting has not previously been published. This study is an early step toward such an analysis.

We found that EPIV catheter indwell time averaged 4 days, with a $72 \%$ success rate. Previous reports of EPIV catheter use in neonates claimed an indwell time of 6 to 10 days, with less than $50 \%$ of the catheters remaining in place until they were no longer needed. ${ }^{1,10-12,25}$ Earlier data, as well as our own, support the conclusion that EPIV catheter use in neonates is more effective than a PIV catheter.

We reported a shorter average EPIV catheter indwell time than previous reports. ${ }^{1,10-12}$ Our experience suggests that we sometimes missed opportunities in maximizing EPIV catheter usage. Dawson ${ }^{10}$ suggested inserting EPIV catheter at the time of admission in all neonates who are expected to need at least 3 days of vascular access; our EPIV catheters were commonly inserted at the end of the treatment course after exhausting multiple PIV catheters, as
$13 \%$ of our EPIV catheters were used for only 2 days or less. We speculated that, in some cases, EPIV catheter can be placed at the beginning of fluid or antibiotic treatment course, which maximizes EPIV catheter's indwell days to the entire time intravenous access is needed. In addition, prior studies included VLBW neonates $(<1500 \mathrm{~g})$, a group that typically requires more time to reach IV nutrition independence than do larger neonates, and thus need longer catheter indwell times.

We reported a much higher success rate than others. At the beginning of our EPIV catheter implementation, placement was not limited on the basis of BW or GA at birth. A high EPIV catheter failure rate $(59 \%, \mathrm{n}=22)$ was found in VLBW neonates, which is comparable with the previously published failure rate in similar population. ${ }^{11}$ Thus, neonates with BW of less than $1500 \mathrm{~g}$ or GA of less than 32 weeks are excluded from our current EPIV catheter protocol. Also, some VLBW neonates require a parenteral nutrition composition and osmolality that is best provided using a PICC. Therefore, our unit considers using PICCs rather than EPIV catheters for VLBW neonates.

We observed a good safety profile of EPIV catheter. Neonates with EPIV catheter had a lower incidence of hyaluronidase-treated IV fluid extravasation than did those with PIV catheters. In addition, we detected no life-threatening complications or catheter infections associated with EPIV catheter. Four cases of PICC-associated life-threatening complications including CLABSI, arrhythmia, superior vena cava obstruction, and peritonitis were noted in this study group.

We recognize weaknesses in our study. First, our EPIV catheter experience involves a single center and is a retrospective analysis and thus lacks the rigor of a prospective randomized device trial. Since EPIV catheter tip is located in a peripheral vessel, its usage is not appropriate for neonates requiring high dextrose concentration, high total parenteral nutrition osmolarity, or medications that should be given only through a central catheter. Neonates who have those needs would be considered for PICC placement instead. Therefore, our EPIV catheter recipients may have been, as a group, somewhat less ill than those in whom a PICC was used. We did not adjust the illness severity as a confounding factor. Also, we lack data on our PIV catheter indwell times and success rates and on the number of attempts per vascular access insertion. Finally, EPIV catheter seems to be less costly than PICC or PIV catheter for 1-week use; however, we recognize that our cost methods are imprecise and incomplete and that the value of any intravenous access varies substantially on a case-bycase basis. In fact, our determination of the cost advantages of EPIV catheter may be understated, because we did not attempt to include expenses of 
Summary of Recommendations for Practice and Research

\begin{tabular}{|c|c|}
\hline What we know: & $\begin{array}{l}\text { - Vascular access is a challenge in the NICU. } \\
\text { - EPIV catheters are 6-cm and 8-cm silicone catheters inserted into neonatal } \\
\text { peripheral veins. Similar devices have been safely used in adults but have not } \\
\text { gained popularity in neonates due to a lack of evidence. } \\
\text { - Overall, neonatal PIV catheters do not remain in place for long periods of time } \\
\text { and multiple PIV catheters are replaced prior to the completion of therapy. } \\
\text { - PICCs have longer indwell times than PIV catheters and EPIV catheters but are } \\
\text { expensive and are associated with life-threatening complications. }\end{array}$ \\
\hline What needs to be studied: & $\begin{array}{l}\text { - The efficacy of EPIV catheter within the neonatal population as compared with } \\
\text { other more widely accepted forms of vascular access. } \\
\text { - The safety of EPIV catheter compared with other vascular access devices used by } \\
\text { neonates. } \\
\text { - The cost-effectiveness of EPIV catheter as compared with other vascular access } \\
\text { devices used in the same population. }\end{array}$ \\
\hline What can we do today: & $\begin{array}{l}\text { - Provide a wide variety of vascular access devices for neonates including newer } \\
\text { devices such as EPIV catheter. } \\
\text { - Make informed decisions on which vascular access device to use with each } \\
\text { neonate based on his or her individual needs. } \\
\text { - Aim to increase available literature regarding vascular access within the neonatal } \\
\text { population by gathering and publishing data. }\end{array}$ \\
\hline
\end{tabular}

treating PICC-associated complications or lessening discomfort from repeated PIV catheter punctures.

We conclude that for selected NICU patients, EPIV catheter may have advantages over PIV catheter and PICC. A randomized prospective trial and additional studies are needed to validate the potential value of EPIV catheter usage for neonatal care.

\section{Acknowledgments}

The authors thank Dr Robert Christensen and Dr Roger Faix, Division of Neonatology, University of Utah, for review of the article.

\section{References}

1. Wyckoff MM. Midline catheter use in the premature and full-term infant. J Assoc Vasc Access. 1999:4(3):26-29.

2. Ainsworth S, McGuire W. Percutaneous central venous catheters versus peripheral cannulae for delivery of parenteral nutrition in neonates. Cochrane Database Syst Rev. 2015;(10):CD004219. doi:10.1002/14651858.CD004219.pub4

3. Romesber TL. Evaluating the evidence for midline catheter use in the newborn intensive care unit. J Infus Nurs. 2015;38(6):420-429. doi:10.1097/NAN.0000000000000134.

4. Ramasethu J. Complications of vascular catheters in the neonatal intensive care unit. Clin Perinatol. 2008;35(1):199-222. doi:10.1016/j. clp.2007.11.007

5. Pettit J. Technological advances for PICC placement and management. Adv Neonatal Care. 2007:7(3):122-131.

6. Colacchio K, Deng Y, Northrup V, Bizzarro MJ. Complications associated with central and non-central venous catheters in a neonatal intensive care unit. J Perinatol. 2012;32(12):941-946. doi:10.1038/jp.2012.7.

7. Pettit J. Assessment of infants with peripherally inserted central catheters: part 2. Detecting less frequently occurring complications. Adv Neonatal Care. 2003:3(1):14-26.

8. Nadroo AM, Lin J, Green RS, Magid MS, Holzman IR. Death as a complication of peripherally inserted central catheters in neonates. J Pediatr. 2001;138(4):599-601.

9. Franck LS, Hummel D, Connell K, Quinn D, Montgomery J. The safety and efficacy of peripheral intravenous catheters in ill neonates. Neonatal Netw. 2001;20(5):33-38.
10. Dawson D. Midline catheters in neonatal patients: evaluating a change in practice. J Assoc Vasc Access. 2002;7(2):17-19.

11. Leick-Rude MK, Haney B. Midline catheter use in the intensive care nursery. Neonatal Netw. 2006;25(3):189-199.

12. Lesser E, Chhabra R, Brion LP, Suresh BR. Use of midline catheters in low birth weight infants. J Perinatol. 1996;16(3, pt 1): 205-207.

13. Tobin CR. The Teflon intravenous catheter: incidence of phlebitis and duration of catheter life in the neonatal patient. J Obstet Gynecol Neonatal Nurs. 1988;17(1):35-42. doi:10.1111/j.1552-6909.1988. tb00412.x

14. Smith $A B$, Wilkinson-Faulk $D$. Factors affecting the life span of peripheral intravenous lines in hospitalized infants. Pediatr Nurs. 1994;20(6):543-547.

15. McCullen KL, Peiper B. A retrospective chart review of risk factors for extravasation among neonates receiving peripheral intravascular fluids. J Wound Ostomy Continence Nurs. 2006;33(2):133-139.

16. Restieaux M, Maw A, Broadbent $R$, Jackson P, Barker D, Wheeler $B$. Neonatal extravasation injury: prevention and management in Australia and New Zealand-a survey of current practice. BMC Pediatr. 2013:13:34. doi:11.1186/1471-2431-13-34.

17. Yan Y-M, Fan Q-L, Li A-Q, Chen J-L, Dong F-F, Gong M. Treatment of cutaneous injuries of neonates induced by drug extravasation with hyaluronidase and hirudoid. Iranian J Pediatr. 2014;24(4):352358.

18. Alexandrou E, Jamjan LM, Spencer T, Forst SA, Salamonson DP. The use of midline catheters in the adult acute care setting - clinical implications and recommendation for practice. J Assoc Vasc Access. 2011;16(1):35-41

19. Wood M. Improving patient comfort by increasing completion of therapy rates with fewer risks for patients through the use of midline catheters. J Assoc Vasc Access. 2015;20(4):250. doi:10.1016/j. java.2015.10.043.

20. Moran MA. Introducing the midline catheter as a new intravenous access device in neonates. Neonatal Intensive Care. 1992;5(3):36-42.

21. NeoMagic [product brochure]. Sparks, NV: Neo Medical Inc; 2011

22. Gorski L, Hadaway L, Hagle ME, McGoldrick M, Orr M, Doellman D. Infusion therapy: standards of practice. J Infus Nurs. 2016;39(1S): S52.

23. Clark E, Giambra B, Hingl J, Doellman D, Tofani B, Johnson N Reducing risk of harm from extravasation: a 3-tiered evidence-based list of pediatric peripheral intravenous infusates. J Infus Nurs. 2013:36(1):37-45. doi:10.1097/NAN.0b013e3182798844.

24. Anderson NR. Midline catheters: the middle ground of intravenous therapy administration. J Infus Nurs. 2004;27(5):313-321.

25. Reynolds J. Comparison of percutaneous venous catheters and Teflon catheters for intravenous therapy in neonates. Neonatal Netw. 1993;12(5):33-39. 